\title{
Quantum dots as biological optical imaging tools
}

\begin{abstract}
Quantum dots are semiconducting nanocrystals having dimensions in the range of 2-10 nanometers that have attracted for their clinical and diagnostic applications. Their surface modification or functionalization has been in significant progress over last decades to make them precise nanoprobes for biological imaging. This practice is carried out with bioconjugation of prepared quantum dots in to various biological/ chemical molecules with broad absorption spectra, bright emission and photophysical robustness. This surface modified quantum dots might be further improved tool for brain targeted or cell imaging for getting accurate localization of tumor or diseased tissues to achieve effective diagnostic biopsy and complete surgical resection for improved surgical management of brain or cell tumors as improved optical imaging and optical spectroscopy tools.
\end{abstract}

Keywords: quantum dots, nanoparticles, brain imaging, optical imaging tools, bioconjugation, tumor, polyethylene glycol
Volume 4 Issue I - 2017

Kirti Rani
Amity Institute of Biotechnology, India

Correspondence: Dr Kirti Rani,Assistant Professor (III), Amity Institute of Biotechnology, Amity University Uttar Pradesh, Noida, Sec-125, Gautam Buddha Nagar, Noida-201313 (UP), India, Tel +9|- | 20-4392946, +91-9990329492,

Email krsharma@amity.edu, Kirtisharma2k@rediffmail.com

Received: April 26, 2017| Published: September 22, 2017

\section{Introduction}

Quantum dots (QDs) are semiconducting nanosized particles material having dimensions in the range of 2-10 nanometers which were first discovered in 1980. In addition, very tiny nanosized QDs can be easily incorporated into a cellular membrane that have low toxicity and possess an intrinsic sensitivity to surrounding electric fields. $^{1-3}$ Quantum dots (QDs) have been subjected for optical imaging as effective nanoscale semiconductors which have unique photophysical properties. ${ }^{4}$ The application of quantum dots (QDs) have been considered for imaging for neuronal action potentials to achieve better resolution imaging tool to analysis brain cell function. ${ }^{5,6}$ The intraoperative diagnosis of brain tumors or diseased cells and accurate evaluation of their biomarkers can be improved diagnostic to prepare precise guidelines for arranging rapid adjunctive clinical and biomedical studies. This was evaluated for specificity of using of streptavidin-coated conjugated anti-EGFR antibodies quantum dot-labeled antibodies quantum dots (QDs) for rapid visualization of epidermal growth factor receptor (EGFR) expression in human brain tumor cells and human glioma tumor cell lines. ${ }^{7}$

Previously, semiconductor and fluorescent quantum dots (QDs) were considered improved optical imaging tools with their surface modifications for surgical management of brain tumors. The intravenous injection of quantum dots was used with reticuloendothelial system and macrophage sequestration for getting improved brain imaging mapping for tracking the optically labeling the tumors. So, macrophage-mediated delivery of quantum dots to brain tumors might be novel biomedical technique to label tumors or diseased preoperatively through which tumors or any type of diseased cells or tissues may be detected with optical imaging to provide the surgeon with real-time optical feedback during the resection and biopsy of brain tumors or diseased cells/tissues. ${ }^{8}$

Surface modification and functionalization of quantum dots (QDs) may improve their water solubility via using the organometallic processes which protect them from any kind degradation and fluorescence quenching. In addition, their surface modification with polyethylene glycol (PEG) was found to minimize their non- specific uptake in normal organs and provide functional groups for further choosing any bioconjugation process. ${ }^{9}$ Delivery of imaging agents or vectors to the brain is very important for the accurate diagnosis and treatment of various central nervous system (CNS) disorders or neurodegenerative diseases to examine their respective pathophysiology. Optically active modified quantum dots (QDs) might be prove more promising nano-probe with unique physical, chemical, and optical properties for molecular and cellular imaging apart their poor stability and low blood-brain barrier permeability. So, recently, quantum dots (QDs) based cell or biological optical imaging concept was developed for brain and cell imaging by incorporating quantum dots (QDs) into the core of poly (ethylene glycol)-poly(lactic acid) nanoparticles with their surface fabrication with wheat germ agglutinin which were further delivered into the brain or cells via nasal administration route. The resulting surface modified quantum dots (QDs) were found to notices to have increased payload capacity, better aqueous solubility and more safe brain or cells imaging properties. $^{10}$

\section{Conclusion}

The short and precise review article can helpful to provide instant layout to describe the application of quantum dots (QDs) in brain and cell imaging as more efficient optical tools as well as to make them more multifunctional nanoprobes by employing various surface modifications and bioconjugation processes. So, use of functionalized quantum dots (QDs) can be considered more promising nano-labeledprobes for improving their applicability in brain and cell imaging to treat many neurodegenerative or central nervous system disorders and tumors.

\section{Acknowledgements}

I would like also to express my cordially appreciation to Amity University Uttar Pradesh, Noida (INDIA).

\section{Conflict of interest}

The author declares no conflict of interest. 


\section{References}

1. Agarwal R, Domowicz MS, Schwartz NB, et al. Delivery and tracking of quantum dot peptide bioconjugates in an intact developing avian brain. ACS Chem Neurosci. 2015;6(3):494-504.

2. Rowland Clare E, Susumu Kimihiro, Stewart Michael H, et al. Imaging cellular membrane potential through ionization of quantum dots. Proceedings of the SPIE. 2016;9722:1-8.

3. Tsytsarev V, Liao LD, Kong KV, et al. Recent progress in voltage-sensitive dye imaging for neuroscience. J Nanosci Nanotechnol. 2014;14(7):4733-4744

4. Alison Abbott. Neuroscience: Solving the brain. Nature. 2013;499:272274.

5. Empedocles SA, Bawendi MG. Quantum-confined stark effect in single CdSe nanocrystallite quantum dots. Science. 1997;278(5346):2114 2117 .
6. Rowland CE, Susumu K, Stewart MH, et al. Electric field modulation of semiconductor quantum dot photoluminescence: insights into the design of robust voltage-sensitive cellular imaging probes. Nano Lett. 2015;15(10):6848-6854.

7. Wang J, Yong WH, Sun Y, et al. Receptor-targeted quantum dots: fluorescent probes for brain tumor diagnosis. $J$ Biomed Opt. 2007;12(4):044021.

8. Popescu MA, Toms SA. In vivo optical imaging using quantum dots for the management of brain tumors. Expert Rev Mol Diagn. 2006;6(6):879-890.

9. Schipper ML, Iyer G, Koh AL, et al. Particle size, surface coating, and PEGylation influence the biodistribution of quantum dots in living mice. Small. 2009;5(1):126-134.

10. Gao X, Chen J, Wu B, et al. Quantum dots bearing lectin-functionalized nanoparticles as a platform for In Vivo brain imaging. Bioconjug Chem. 2008;19(11):2189-2195. 